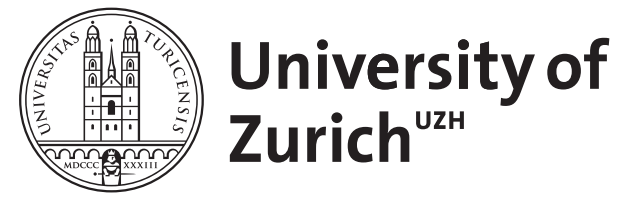

\title{
Biomechanik in Traumatologie und Rechtsmedizin
}

Niederer, Peter F

DOI: https://doi.org/10.1515/bmte.1993.38.s1.21

Posted at the Zurich Open Repository and Archive, University of Zurich ZORA URL: https://doi.org/10.5167/uzh-155343

Journal Article

Published Version

Originally published at:

Niederer, Peter F (1993). Biomechanik in Traumatologie und Rechtsmedizin. Biomedizinische Technik. Biomedical engineering, 38(s1):21-26.

DOI: https://doi.org/10.1515/bmte.1993.38.s1.21 


\title{
Biomechanik in Traumatologie und Rechtsmedizin
}

\author{
Peter F. Niederer \\ Institut für biomedizinische Technik und medizinische Informatik \\ Universităt und ETH Zürich
}

\section{Einleitung}

Die Biomechanik befasst sich mit der Art und Intensităt der Wirkung von Krăften auf biologische Objekte. Die Wirkung kann sich dabei auf den Bewegungs- und/oder den Deformationszustand eines Korpers beziehen.

Eine besondere Situation liegt vor, wenn die Krăfte (mindestens kurzzeitig) irreversible Schädigungen am betrachteten Objekt hervorrufen (längerfristig verlaufende Heilungsprozesse und Langzeitschäden werden hier nicht mitberücksichtigt). Die Untersuchung solcher Vorgănge geschieht im Rahmen der Traumabiomechanik. Naturgemäss ist die Rechtsmedizin (forensische Medizin) in besonders starkem Ausmasse an Fragen der Traumabiomechanik interessiert.

Von Bedeutung ist in diesem Zusammenhang der Umstand, dass bis zum Alter von 45 Jahren Verletzungen, insbesondere als Folge von Verkehrsunfällen die häufigste Todesursache darstellen. Der durch Verletzungen hervorgerufene volkswirtschaftliche Schaden, berechnet als Verlust an produktiven Lebensjahren (etwas willkürlich definiert als Komplement bis zum 65. Altersjahr), ist grösser als der durch irgendwelche Krankheiten verursachte Verlust [1]. Entsprechend kommt der Unfall- und Verletzungsprävention entscheidende Bedeutung zu, wobei allerdings zu beachten ist, dass viele Aspekte in diesem Bereich nicht physikalischtechnischer Natur sind (z. B. risikoreiches Autofahren, Kriminalität).

Grundlage sowohl der Unfallprävention als auch der forensischen Beurteilung eines Falles ist eine Dokumentation des Vorgefallenen sowie eine darauf basierende Rekonstruktion. Notwendig ist sodann eine Kenntnis von Verletzungsmechanismen und der entsprechenden biomechanischen Toleranzen. Dabei ist darauf hinzuweisen dass in diesem Bereich die biologische Variabilităt besonders stark zum Ausdruck kommt: Beispielsweise variiert die Bruchlast des Oberschenkelhalses zwischen einigen $\mathrm{kN}$ bei gesunden Jungen bis zu unter Umstănden praktisch Null im Alter, wo bei stark osteoporotischen Personen Spontanbrüche auftreten können.

\section{Methoden}

Traumabiomechanische Untersuchungen an Menschen sind unter physiologischen Bedingungen grundsătzlich nicht möglich. Man ist deshalb auf indirekte Methoden angewiesen. Diese können in drei Gruppen unterteilt werden:

Rekonstruktion realer Unfälle: Mit Hilfe zuverlässiger Rekonstruktionsmethoden wird angestrebt, aus realen Unfallen Erkenntnisse bezüglich Verletzungsmechanismen und biomechanischer Toleranzen zu gewinnen. Voraussetzung dabei ist eine genaue und vollstăndige Dokumentation des Vorgefallenen vor Ort [2], was in der Regel nur durch speziell ausgebildete Polizeiorgane erfolgen kann. Der Zusammenarbeit von Forschungsgruppen mit Polizeidienststellen kommt entsprechend grosse Bedeutung zu. Dabei entsteht ein oft unterschätzter zusätzlicher Vorteil, indem der zunăchst grössere Aufwand bei der Dokumentation (Arbeitszeit von Polizeibeamten, Absperren des Verkehrs, etc.) durch eventuelles Vermeiden von späteren jahrelangen Rechtsstreitigkeiten mit hăufig nutzlosen Gutachten (wegen Fehlens relevanter primärer Information) bei weitem mehr als wettgemacht wird.

Experimente mit Surrogaten: Anthropomorphe Messpuppen eignen sich für die Simulation von 
Bewegungsablăufen. Aus daran gemessenen Krăften und Beschleunigungen werden gelegentlich gewisse Schutzkriterien abgeleitet (beispielsweise das Head Injury Criterion [3]). Obwohl die Einhaltung solcher Kriterien zum Teil gesetzlich verankert ist (z. B. FMVSS 208), ist deren Relevanz jedoch umstritten. Wegen unterschiedlicher biomechanischer Konstitution zwischen Mensch und Tier sind sodann Tierversuche in der Unfallforschung kaum von Bedeutung. Eine Ausnahme bildet die orthopädische Traumatologie, wo der Tierversuch für die Implantatentwicklung wichtig ist. Für spezielle Fragestellungen werden schliesslich auch Versuche an Leichen durchgeführt.

Mathematische Modellierung: Für die Zwecke der Bewegungsanalyse hat es sich erwiesen, dass der menschliche Körper, ähnlich wie bei Messpuppen, als System starrer Teilkörper modelliert werden kann. Dabei entstehen Systeme von gewöhnlichen Differentialgleichungen, deren Anzahl dem Freiheitsgrad des Systems entspricht. Für die Untersuchung von Verletzungsvorgängen sind dagegen kontinuumsmechanische Ansätze zu verwenden, d.h. es müssen partielle Differentialgleichungen formuliert werden. $\mathrm{Zu}$ deren Lösung eignet sich u.a. die Methode der Finiten Elemente. Das Problem besteht darin, dass nichtlineares viskoelastisches Stoffverhalten von inhomogenen Körpern bei grossen Deformationen zu modellieren ist. Die dafür benötigten Stoffgesetze und Parameterwerte sind grösstenteils wenig bekannt.

\section{Beispiele}

\subsection{Bewegungsanalyse}

Fallweite bet Fenstersturz: Ein 50-jähriger Mann fiel aus dem Fenster seines Wohnhauses aus $8 \mathrm{~m}$ Höhe auf den Boden, wobei er sich tödliche Verletzungen zuzog. Die Aufprallstelle am Boden befand sich in einem Abstand von $2.70 \mathrm{~m}$ von der Hausmauer entfernt. Die Frage erhob sich, ob eine solche Fallweite mit einem passiv verlaufenden Fall aus dem Fenster vereinbar war (unter diesen Umstănden hătte es sich um einen Unfall gehandelt), oder ob notwendigerweise ein aktiver Sprung angenommen werden musste (dies bedeutete Selbstmord; eine kriminelle Handlung konnte ausgeschlossen werden). Aus versicherungstechnischen Grunden ergaben sich je nach Fall enorme finanzielle Konsequenzen. Die mathematische Analyse zeigle, dass bei einem unfallmässigen Sturz rückwarts aus dem Fenster mit
Hochwerfen der Arme als Schreckreaktion (Figur 1) eine Fallweite von $2.70 \mathrm{~m}$ theoretisch knapp erreicht werden konnte. In mehreren Sprung- und Fallversuchen wurde dieser Umstand bestätigt. Vom physikalischen Standpunkt war ein aktiver Sprung, d.h. ein Selbstmord in jedem Falle möglich, da die fragliche Fall- (Sprung-) Weite dabei problemlos erreicht wird.
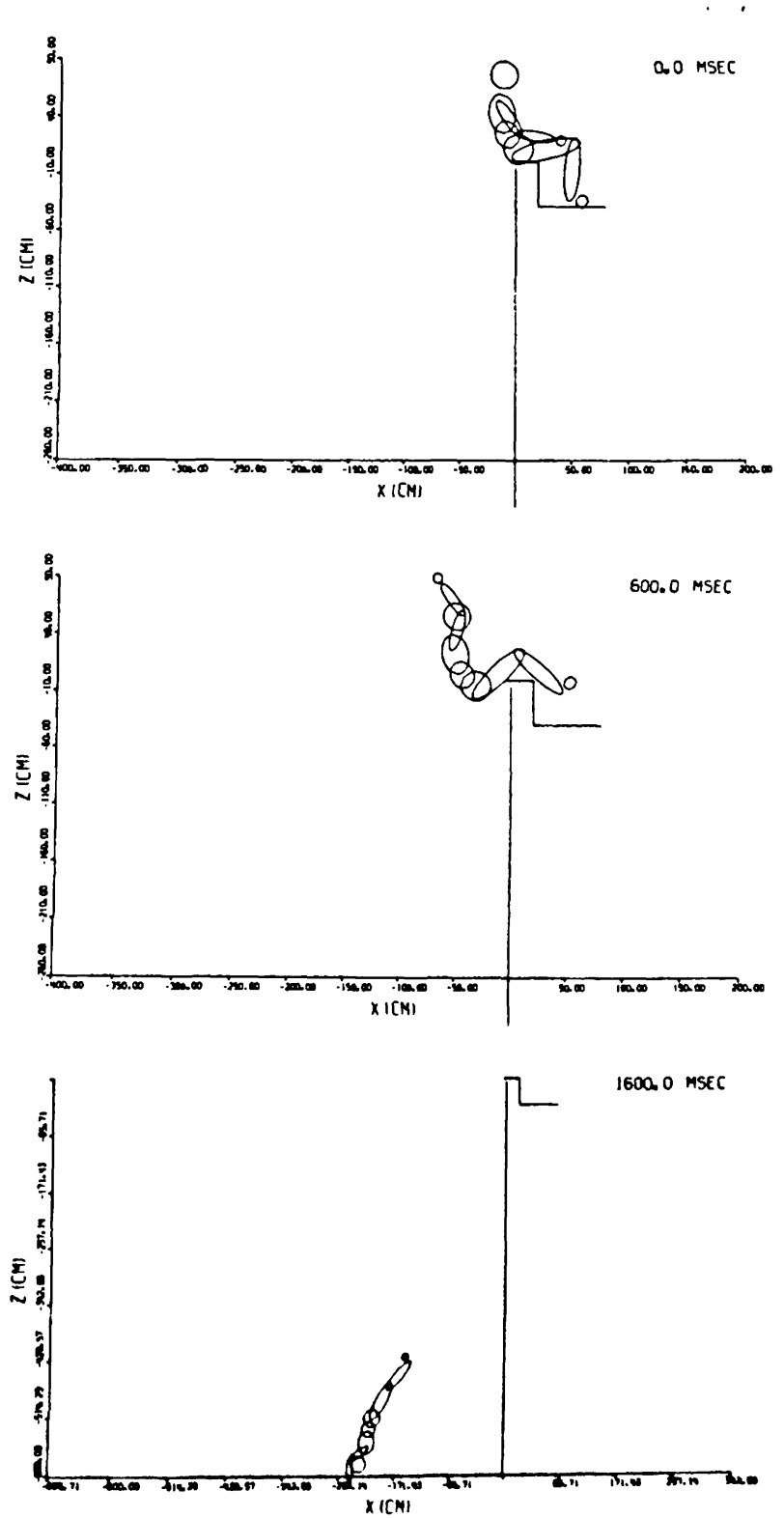

Figur 1: Unfallmassiger Sturz ruchwarts aus cinem Fenster mit Hochwerfen der Arme als Schreckreaktion. Dabei wird dem Körper ein zusatzlicher Impuls in horizontaler Richtung verliehen, aufgrund dessen sich die Fallweite irrgrossert.

\section{Biomedizinische Technik Band 38 • Ergănzungsband • 1993}


Auto-Fussganger-Koollision: Ein Fussganger wurde beim Uberqueren der Strasse bei Regen von einem Auto crfasst und getotet (Situationsplan Figur 2a: [1]: Mutmassliche Gehrichtung des Fussgängers, [2]: Endlage, [3]: Wegen regennasser Strasse nur tcilwcise sichtbare Blockierspuren). Der Fahrer sagte aus, dic zulăssige Hochstgeschwindigkeit von $\mathbf{6 0}$ $\mathrm{km} / \mathrm{h}$ nicht uberschritten zu haben.
Dank aufmerksamer Unfallprotokollierung durch die Polizeibeamten konnte ein Fussabdruck des Fussgăngers auf der Kofferraumabdeckung dokumentiert werden ([4] dabei befand sich die Endlage des Fussgangers vor dem Autol). Ein zugehöriger Bewegungsablauf ist jedoch bei Kollisionsgeschwindigkeiten bis zu $60 \mathrm{~km} / \mathrm{h}$ nicht moglich (Figur 2b).

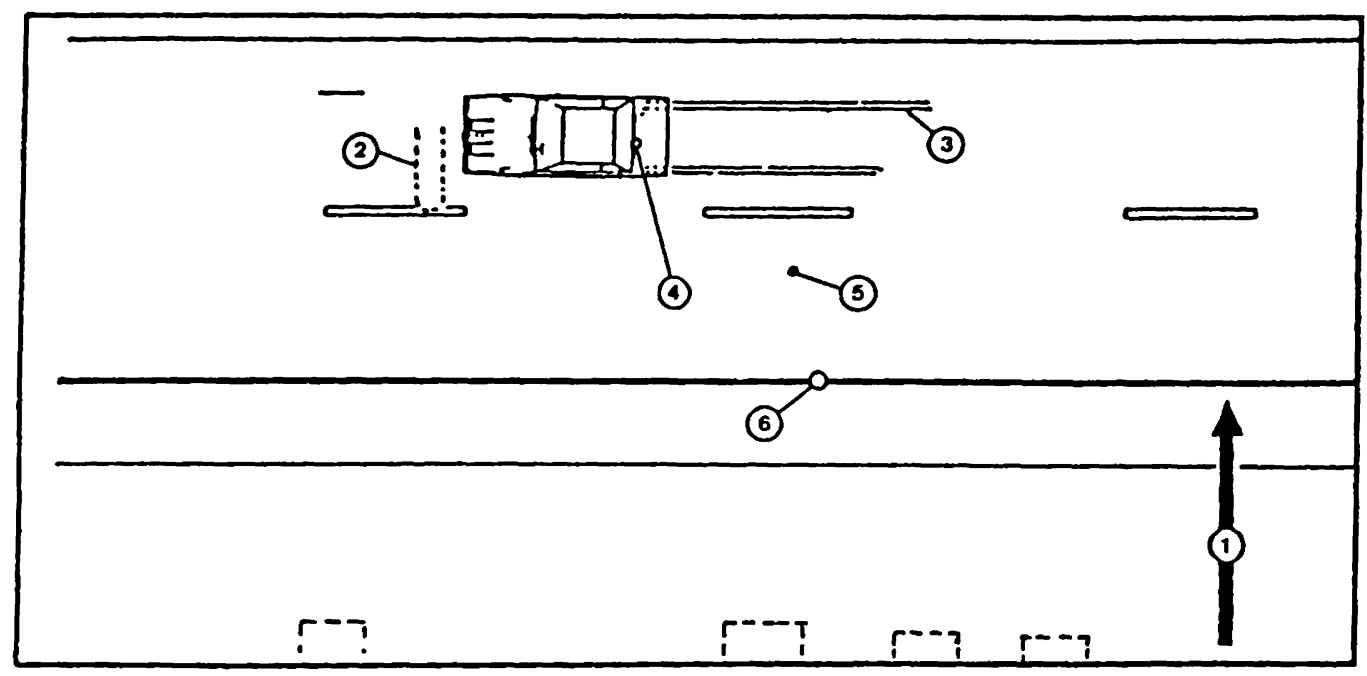

Figur 2a: Fahrzeug-Fussgangerkollision (siehe Text). Bei regennasser Fahrbahn entsteht keine bezuglich Fahrgeschwindigkeit auswertbare Blockierspur.
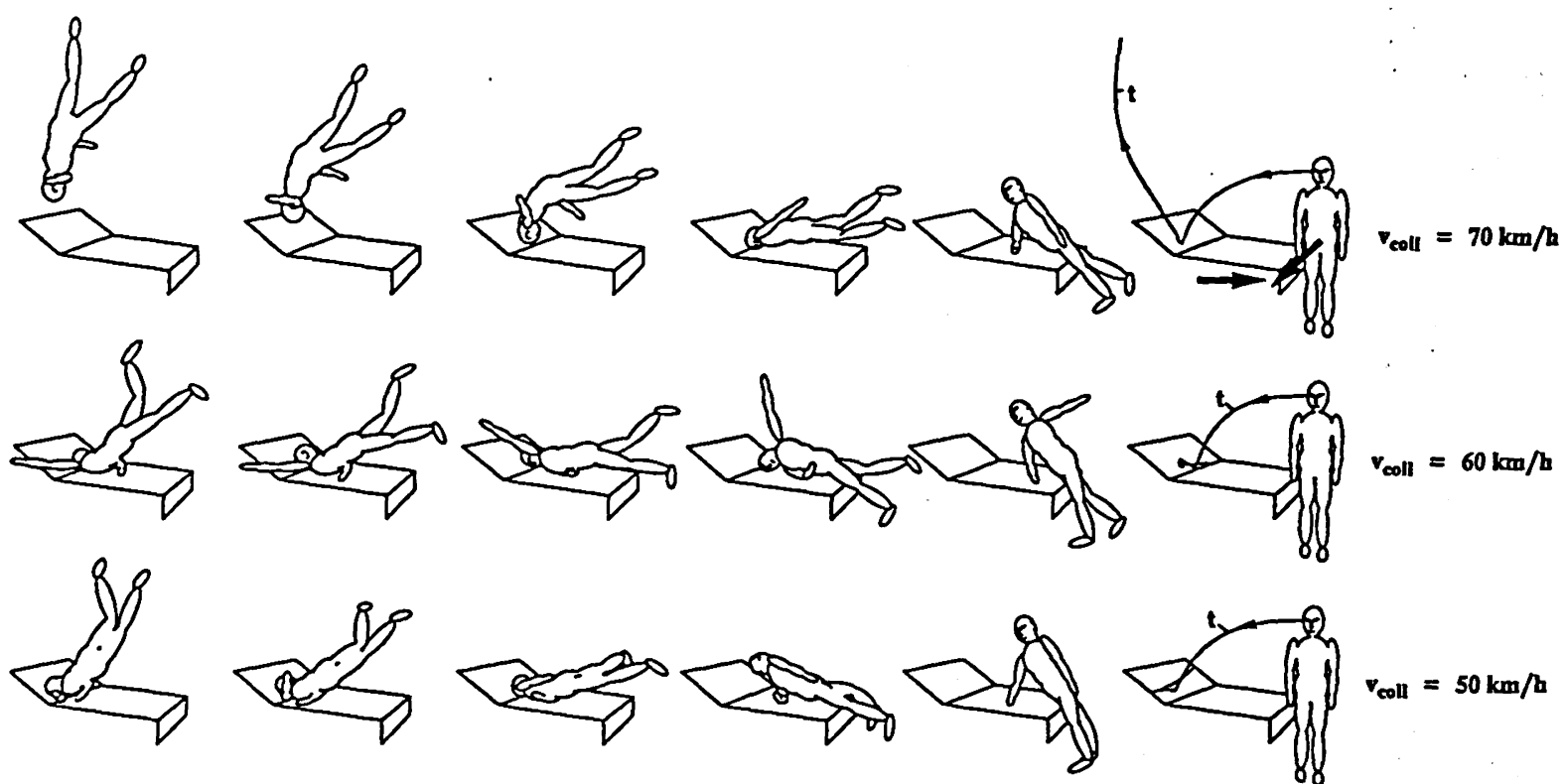

$250 \mathrm{msec}$

$200 \mathrm{msec}$

$150 \mathrm{msec}$

$100 \mathrm{msec}$

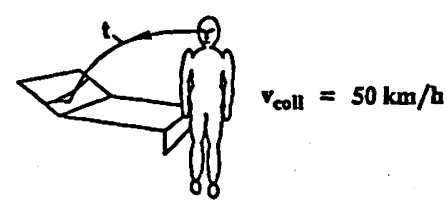

0 msec

Figur 2b: Mathematisches Modell des in der Figur $2 a$ dokumentierten Anpralles. Bei einer Kollisionsgeschwindigkeit von $70 \mathrm{~km} / \mathrm{h}$ entsteht ein Ueberschlag des Fussgängers mit anschliessender Unterfahrung durch das Fahrzeug ( $t$ : Trajektorie des Massenmittelpunktes des Kopfes relativ zum Fahrzeug). Bei geringeren Kollisionsgeschwindigkeiten verbleibt die Trajektorie im Bereich der Fronthaube (Computermodell Calspan CVS [6]). 
eigentlichen Angriffes des Tăters mit der Absicht, dem Opfer Verletzungen zuzuruhren (nachweisbar beispiclswcise bei Vorhandenscin mehrerer Stichwunden), bzw. eines passiven Verhaltens des Taters (of bchauptetes Hincinlaufen oder -fallen des Opfers in das Mcsser), was rechtlich unterschiedlich gewertet wird.

\section{Schlussbemerkungen}

Forschung und Entwicklung auf dem Gebiet der der forensischen Traumabiomechanik (in Abgrenzung zur orthopädisch orientierten Traumabiomechanik) konzentriert sich grob auf vier Bereiche: Erstens sollen bessere Surrogate (Messpuppen) realistischere Messergebnisse ergeben. Weiterhin sind die biomechanischen Toleranzen besser zu definieren, wobei auch dem Problem des Populationsmittelwertes vermehrt Beachtung geschenkt werden muss. $\mathrm{Da}$ sich die biomechanische Konstitution in Abhängigkeit des Alters stark åndert, ist es fraglich, ob die Angabe globaler Werte sinnvoll ist.

Wie bereits darauf hingewiesen wurde, sind traumabiomechanische Untersuchungen am Menschen nicht möglich. Besondere Bedeutung hat deshalb drittens die Methode der Finiten Elemente, deren Anwendung heute auch bei nichtlinearen viskoelastischen Problemen realistische Resultate liefert, falls das Materialverhalten genügend gut beschrieben werden kann. Viertens sind Methoden der rechnergestützten Simulation und Animation allgemein von rasch zunehmender Bedeutung, und die Technik der Virtuellen Realităt wird sich nebst der Darstellung von Unfallereignissen im forensischen Umfeld auch für Ausbildungszswecke eignen.

\section{Literatur}

[1] National Research Council: Injury in America. Washington 1983.

[2] Niederer P.: The Accuracy and Reliability of Accident Reconstruction. In: Automotive Engineering and Litigation (G. Peters and B. Peters, eds.), John Wiley and Sons, NY 1991.
[3] Backaitis S.: The Head Injury Criterion. In: Head and Neck Injury Criteria. A Consensus Workshop. NHTSA Washington 1981.

[4] Jenzer G., Walz F.: Die "Schwere" des "Schleudertraumas" der HWS. Z. Unfallchir. Vers. med. 84 (1991) 7.

[5] Kaatsch H.-J., Mehrens C., Nietert M.: Der reproduzierbare Messerstich. Rechtsmedizin 3 (1993) 67.

[6] Fleck J., Butler F., Vogel S.: An Improved Three-Dimensional Computer Simulation of Vehicle Crash Victims. US Dept. of Transportation, DOT HS-801507 (1973). 


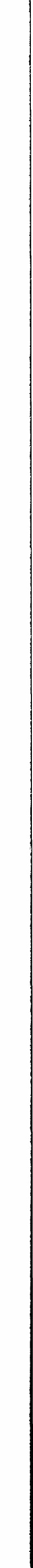

